\title{
DRUG-RELATED PROBLEMS IN HOSPITALIZED GERIATRIC PATIENTS WITH DIABETES MELLITUS
}

\author{
DEBBY DYSTRA MAHARANI ${ }^{1}$, NADIA FARHANAH SYAFHAN ${ }^{1 *}$, YETTI HERSUNARYATI ${ }^{2}$ \\ ${ }^{1}$ Department of Pharmacy, Faculty of Pharmacy, Universitas Indonesia, Depok, 16424, Indonesia. ${ }^{2}$ Department of Pharmacy, Pharmacy \\ Installation, Gatot Soebroto Army Hospital, Jakarta, 10410, Indonesia. Email: nadia.farhanah@farmasi.ui.ac.id
}

Received: 31 May 2018, Revised and Accepted: 22 November 2018

\section{ABSTRACT}

Objective: This study aimed at analyzing drug-related problems (DRPs) in hospitalized geriatric patients with diabetes mellitus.

Methods: We prospectively collected data of 26 hospitalized geriatric patients at the Gatot Soebroto Army Hospital (age, $>60$ year) who underwent inpatient treatment for at least 1 month between February and April 2015. Readable data were obtained from prescriptions, medical records, and index card/nurses records. We obtained data on 299 drug treatments for 26 patients and identified 166 DRPs. These were analyzed based on the Pharmaceutical Care Network Europe Version 6.2.

Results: The percentage of treatment effectiveness (50.6\%) and adverse drug reactions (49.4\%) was predominantly due to an inappropriate drugdrug or drug-food combination, including the incidence of drug interactions $(20.4 \%)$.

Keywords: Adverse reaction, Drug-related problem, Gatot Soebroto Army Hospital, Geriatric, Treatment effectiveness.

(C) 2018 The Authors. Published by Innovare Academic Sciences Pvt Ltd. This is an open access article under the CC BY license (http://creativecommons. org/licenses/by/4. 0/) DOI: http://dx.doi.org/10.22159/ijap.2018.v10s1.30

\section{INTRODUCTION}

Indonesia ranks $4^{\text {th }}$ among the countries with the largest number of diabetes mellitus (DM) cases worldwide, after India, China, and US, based on information obtained from the World Health Organization in 2006. In 2009, the International Diabetes Federation predicted an increase in the number of individuals with DM in Indonesia from 7 to 12 million by 2030. Currently, there are two therapeutic approaches for individuals with DM - with or without drugs. Treatment without drugs is based on specific dietary guidelines, known as nutritional therapy, and physical activity. If this approach does not lead to the therapeutic goal, then drugs are introduced. Specifically, pharmacological steps can be in the form of insulin therapy, oral hypoglycemic drug therapy, or a combination of both [1-3]. Insulin is the treatment for patients with type $1 \mathrm{DM}$, whereas oral hypoglycemic drugs are intended to help in the treatment of patients with type II DM. The management of DM with drug therapy may lead to drug-related problems (DRPs) and an inability to achieve therapeutic goals. Insulin administration may cause hypoglycemia [1,4]. Adverse drug effects are a subset of DRP issues.

DRP is a condition associated with drug therapy that may potentially interfere with the desired health clinical outcomes [5]. Research conducted in French hospitals has suggested that frequent DRP is the administration of drugs contraindicated with patients' condition (21.3\%), improper administration (20.6\%), subtherapeutic dose $(19.2 \%)$, and drug interactions (12.6\%). An Indonesian study has shown that $78.2 \%$ of geriatric patients experienced DRP during hospitalization [6]. Older adults are particularly vulnerable to DRP for the following two main reasons: (1) Age-related physiological changes may alter the pharmacokinetic and pharmacodynamic properties of the drug and (2) the presence of comorbidities and the use of some drugs referred as polypharmacy. Polypharmacy relates to the use of multiple medications and treatments through various administrative routes exceeding clinical indications [7-10].

There are several classifications of DRP, including those according to Strand, Granada II, Pharmaceutical Care Network Europe (PCNE), and Apoteket AB. PCNE classifies DRP based on major domains and subdomains in detail [11]. The latest PCNE classification is a revised 2010 known as "the classification PCNE V6.2." PCNE V6.2 has been unveiled by the Ministry of Health as a guide for DRP identification in current guidelines for pharmacists [12]. Gatot Soebroto Army Hospital is a Class A hospital owned by the Government of the Republic of Indonesia. Gatot Soebroto Army Hospital has medical services specialized in internal disease providing care for metabolic endocrine geriatric/diabetes. This study aimed at analyzing DRPs in geriatric patients with DM since Gatot Soebroto Army Hospital serves a high number of geriatric patients with DM.

\section{METHODS}

We performed a descriptive analytic research. Data were prospectively collected. Specifically, we observed secondary data in the form of drug prescriptions, medical records, and cardiac/nurse records. Data collected in this study originated from geriatric inpatients with DM treated at the Gatot Soebroto Army Hospital between February and April 2015.

Data matching the inclusion criteria were collected either through the pharmacy (for inpatient prescriptions) or through the hospital administration and registration office (medical record number and cardiac/nurse notes). DRP was analyzed using available literature, including Pharmacotherapy A Pathophysiologic Approach, $7^{\text {th }}$ edition; Drug Information Handbook, $17^{\text {th }}$ edition; Geriatric Dosage Handbook in 2005; Journal of Pharmacy of Clinic; MIMS; and ISO 2014. In addition, a specialized search in drug interaction was conducted using software Micromedex $^{\circledR}$.

Patients' data collected included the name, medical record number, age, diagnosis, complaints, prescribed medications, dosage regimen, and treatment duration. Data were reported on pre-prepared data collection sheets. We identified the presence of DRP by filling out a list of contents based on the PCNE V6.2 referring to the literature with the main domains of therapeutic effectiveness and adverse drug reactions (ADRs). 


\section{RESULTS}

\section{Characteristics of research sample}

Data were obtained from observation of patients' medical records. Collected information included sex, DM type, hospital length, history of DM, and DM complications. Characteristics of the study group are shown in Table 1.

\section{DRPs in geriatric patients with DM}

Data were prospectively collected with the total sampling method. Of the total population evaluated, 26 individuals were found to meet the inclusion criteria. Post-analysis, we observed that all the patients evaluated had DRP. Specifically, $55 \%$ had an actual problem and $45 \%$ had a potential problem. Among the 299 drug therapies underwent by the 26 patients, 166 DRPs were identified. Importantly, among DRPs, therapeutic effectiveness problems had a higher frequency than ADRs (50.6\% vs. $49.4 \%$, respectively; Table 2 and Fig. 1). Percentages of treatment effectiveness and ADR subdomain problems are shown in Figs. 2 and 3.

\section{DISCUSSION}

\section{Therapeutic effectiveness problems}

Ineffective medication or treatment failure

The number of cases with ineffective drug or failed treatment amounted to 37. Possible causes were the use of a lower amount of drug than prescribed (underused), drug administration occurred at a lower frequency than prescribed (under-administered), inappropriate drug selection, incorrect combination of drug-drug and drug-food leading to drug interactions, low doses, and patients' deterioration/improvement requiring dose adjustment.

Ceftriaxone was either underused or under-administered. Specifically, ceftriaxone was to be administered $1 \times 2 \mathrm{~g}$ for 5 day. However, in three patients, the antibiotic was not administered as prescribed. In one of the patients, this resulted in a foot infection that did not improve. Due to the length of obtaining results from a culture ( 5 days), empirical antibiotics are given as initial treatment before culture results are obtained. These antibiotics should be changed if the results of the bacterial culture show resistance to the empirical antibiotics. However, despite the laboratory



Fig. 1: Proportions of primary domain problems (Pharmaceutical Care Network Europe V6.2) ( $n=166)$ results, antibiotic replacement does not take place in some cases. Patients' condition did not improve following the empirical antibiotic probably because of unavailability or high cost of an appropriate antibiotic, or to the common knowledge that in most cases, empirical antibiotics are sufficient to treat the patients' condition.

To date, standardized guidelines on empirical antibiotics' use are unavailable. Patients treated with empirical antibiotics should be evaluated for 48-72 h. In case of improvement, antibiotics should be administered until the patient shows a good clinical response and discontinued after 7 days [13].

Treatment failure because of drug interaction occurred as a result of the concurrent administration of aspirin-valsartan (salicylate-angiotensin II receptor blocker). Specifically, the interaction causes a decrease of valsartan's antihypertensive effect so that the patients' blood pressure continues between systole (140 and $159 \mathrm{mmHg}$ ) and diastole (90$99 \mathrm{mmHg}$ ).

Table 1: Characteristics of the study group $(n=26)$

\begin{tabular}{|c|c|}
\hline Characteristics & n (\%) \\
\hline \multicolumn{2}{|l|}{ Sex } \\
\hline Men & $7(26.9)$ \\
\hline Women & $19(73.1)$ \\
\hline \multicolumn{2}{|l|}{ Type of DM } \\
\hline Type 1 DM & $0(0.0)$ \\
\hline Type 2 DM & $26(100.0)$ \\
\hline \multicolumn{2}{|l|}{ Duration of hospitalization (day) } \\
\hline$\leq 7$ & $16(61.5)$ \\
\hline $8-14$ & $7(26.9)$ \\
\hline$\geq 15$ & $3(11.5)$ \\
\hline \multicolumn{2}{|l|}{ DM history (year) } \\
\hline$\leq 10$ & $4(15.4)$ \\
\hline $11-20$ & $2(7.7)$ \\
\hline $21-30$ & $1(3.8)$ \\
\hline Unknown & $19(73.1)$ \\
\hline \multicolumn{2}{|l|}{ Diabetes complication* } \\
\hline Acute & 12 \\
\hline Diabetic ketoacidosis & $7(26.9)$ \\
\hline Hyperglycemic hyperosmolar status & $0(0.0)$ \\
\hline Hypoglycemic & $5(19.2)$ \\
\hline Chronic & 32 \\
\hline \multicolumn{2}{|l|}{ Macroangiopathy } \\
\hline Heart blood vessels & $3(11.5)$ \\
\hline Peripheral blood vessels & $0(0.0)$ \\
\hline Brain blood vessels & $3(11.5)$ \\
\hline \multicolumn{2}{|l|}{ Microangiopathy } \\
\hline Diabetic retinopathy & $0(0.0)$ \\
\hline Diabetic nephropathy & $7(26.9)$ \\
\hline Neuropathy & $6(23.1)$ \\
\hline Diabetic foot & $6(23.1)$ \\
\hline Hypertension & $7(26.9)$ \\
\hline
\end{tabular}

Description: *0ne patient may experience more than one diabetic complication. DM: Diabetes mellitus

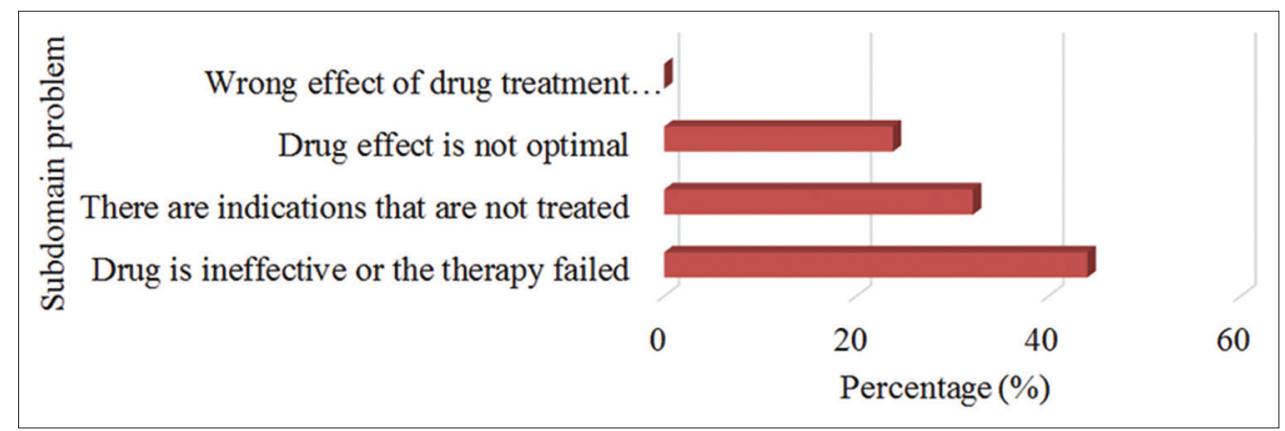

Fig. 2: Percentages of treatment effectiveness subdomain problems (Pharmaceutical Care Network Europe V6.2) (n=84) 
Table 2: Subdomain classification cause (PCNE V6.2) $(\mathrm{n}=181)$

\begin{tabular}{|c|c|c|}
\hline Code & Primary domain & $\begin{array}{l}\text { Number of } \\
\text { problems (\%) }\end{array}$ \\
\hline $\mathrm{C} 1$ & P1. Drug selection & $91(50.3)$ \\
\hline C1.1 & $\begin{array}{l}\text { Selection of inappropriate drugs (not for the most appropriate indications) includes the use of contraindicated } \\
\text { drugs }\end{array}$ & $13(7.2)$ \\
\hline $\mathrm{C} 1.2$ & No indications of drug use or indications of drugs are unclear & $7(3.9)$ \\
\hline C1.3 & Inappropriate drug-food or drug-drug combinations, including in drug interactions & $37(20.4)$ \\
\hline C1.4 & Inappropriate duplication of therapy or inactive ingredients & $0(0.0)$ \\
\hline C1.6 & Many drugs (therapeutic groups or different active ingredients) are prescribed for the same indication & $10(5.5)$ \\
\hline C1.7 & There are more cost-effective drugs available & $0(0.0)$ \\
\hline C1.8 & Synergistic/preventive drugs required are not prescribed & $0(0.0)$ \\
\hline C1.9 & There are newly indicated drugs but have not been prescribed & $18(9.9)$ \\
\hline $\mathrm{C} 2$ & Dosage form selection & $0(0.0)$ \\
\hline $\mathrm{C} 2.1$ & Incorrect dosage form of drugs & $0(0.0)$ \\
\hline $\mathrm{C} 3$ & Dosage selection & $47(26.0)$ \\
\hline C3.1 & The dose of the drug is too low & $9(5.0)$ \\
\hline C3.3 & Dosage settings are infrequent & $7(3.9)$ \\
\hline C3.4 & Dosage settings are too frequent & $10(5.5)$ \\
\hline C3.5 & No blood monitoring was performed & $0(0.0)$ \\
\hline C3.6 & Pharmacokinetic-related issues that require dose adjustment & $8(4.4)$ \\
\hline $\mathrm{C} 3.7$ & Impairment/improvement of sick conditions requiring dose adjustment & $4(2.2)$ \\
\hline $\mathrm{C} 4$ & Duration of treatment determination & $0(0.0)$ \\
\hline C4.1 & Duration of treatment is too short & $0(0.0)$ \\
\hline $\mathrm{C} 4.2$ & Duration of treatment is too long & $0(0.0)$ \\
\hline $\mathrm{C} 5$ & Drug usage process & $41(22.7)$ \\
\hline C5.1 & The timing of drug use or dosage interval is not appropriate & $9(5.0)$ \\
\hline C5.2 & Drug underused/under administered & $22(12.2)$ \\
\hline C5.3 & Drug overused or over administered & $0(0.0)$ \\
\hline C5.4 & Drugs not taken or not administered & $10(5.5)$ \\
\hline C5.5 & Wrong drug taken/administered & $0(0.0)$ \\
\hline C5.6 & Drug abuse (drug use does not conform to official designation) & $0(0.0)$ \\
\hline C5.7 & Patients cannot use drugs or dosage forms according to the rules & $0(0.0)$ \\
\hline $\mathrm{C} 6$ & Logistics (pharmacy) & $1(0.6)$ \\
\hline C6.3 & Drug dispensing error & $0(0.0)$ \\
\hline $\mathrm{C} 7$ & Patient & $1(0.6)$ \\
\hline C7.1 & Patient forgot to drink the medicine & $0(0.0)$ \\
\hline C7.2 & Patients use unnecessary drugs & $0(0.0)$ \\
\hline C7.3 & Patients eat foods that interact with medications & $1(0.6)$ \\
\hline C7.4 & Patient medication storage is not appropriate & $0(0.0)$ \\
\hline $\mathrm{C} 8$ & Others & $0(0.0)$ \\
\hline C8.1 & Other specific causes & $0(0.0)$ \\
\hline $\mathrm{C} 8.2$ & Other unclear causes & $0(0.0)$ \\
\hline
\end{tabular}

Description: One problem domain may comprise more than one cause (maximum three) (PCNE, 2010). PCNE: Pharmaceutical Care Network Europe

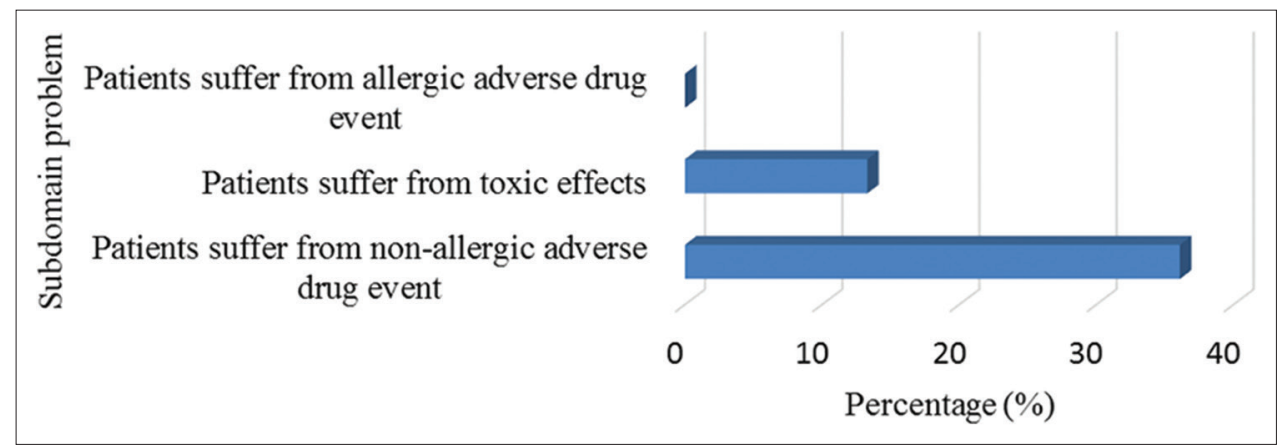

Fig. 3: Percentages of adverse drug reactions subdomain problems (Pharmaceutical Care Network Europe V6.2) (n=82)

An ineffective drug case occurred in a hypertensive patient receiving dose adjustment of candesartan from $1 / 16$ to $1 \times 4$ mg due to improved blood pressure by the previous one measure. Unfortunately, patients' blood pressure increased again in the subsequent checks and there was no dose adjustment (increased dose) for this condition until the end of treatment.
A cause of ineffective drug or failed treatment while on DM medications is the low dose of insulin. In general, geriatric patients who enter a Class A hospital have been previously unsuccessfully treated with oral hypoglycemic drugs, which were substituted by insulin. Before administering a fixed dose of insulin to the patient, insulin is initially provided based on the sliding scale method, with a protocol of blood glucose range on a large scale. 
The commonly used dose of sliding scale has a minimum dose of $4 \mu$ and a maximum dose of $20 \mu / 6 \mathrm{~h}$. This method is used for a maximum of 5 days of treatment. An ineffective scale results in an indeterminate fixed dose of insulin leading to an unclear picture of the patients' blood sugar level (BSL) even until the end of the treatment, resulting in the patients' BSL not improving.

We observed that, occasionally, the sliding scale method was not performed. Instead, the fixed dose method was used, without following treatment guidelines. Specifically, a patient weighed $55 \mathrm{~kg}$ and was given insulin Novorapid ${ }^{\circledR} 3 \times 4 \mu$ in the absence of oral hypoglycemic drugs. The most commonly used type of insulin was aspart (rapidacting, from Novorapid ${ }^{\circledR}$ ). Dosage for both type 1 and type 2 DMs was $0.5-1 \mu \mathrm{kg} /$ day. Therefore, a patient weighing $55 \mathrm{~kg}$ should receive a minimum dose per day of $27.5 \mu$. We observed that BSL in most patients until following checkup was $>200 \mathrm{mg} / \mathrm{dL}$.

\section{Drug effects were not optimal}

In 20 cases, medication effects were not optimal. Specifically, of 20 cases, 14 did not receive DM drug therapy, whereas six received the therapy in the form of insulin. We listed four reasons for the effects of non-optimal drugs, namely low-frequency dosing arrangements, lower dosage than advised in the guidelines (underused), drug administration less frequently than advised in the guidelines (under-administered), and dosage which was too low.

We observed that a suboptimal medication effect in DM therapy is caused by doses of insulin too low. In our case, the patients' BSL improved from before hospital admission but remained high at $\geq 200 \mathrm{mg} / \mathrm{dL}$ until sometime after insulin injections. Non-DM drugs with potentially ineffective drug effects are histamine $\mathrm{H} 2$ antagonist, vitamins, proton-pump inhibitors, NSAIDs, angiotensin-convertingenzyme (ACE) inhibitors, antiemetics, and antibiotics generally caused by drug underuse, under-administration, and infrequent dosing arrangements.

Ranitidine injection (histamine $\mathrm{H} 2$ antagonist) effect is suboptimal due to infrequent dosage regulation. Ranitidine dose regimen prescribed was $2 \times 50 \mathrm{mg}$ i.v.; however, $50 \mathrm{mg}$ of the drug should be administered every $6-8 \mathrm{~h}$ for patients not suffering from kidney disease.

The effect of metoclopramide as antiemetics was suboptimal due to their underuse or underadministration. Specifically, metoclopramide was prescribed at the dosage of $2 \times 1$ tablet according to records we observed. On the contrary, patients with nausea are usually prescribed at the dosage of $3 \times 1$ tablet. As a consequence, after 3 days of treatment, nausea complaints were not reduced.

\section{Effects of inappropriate drug (idiosyncrasies)}

Idiosyncrasy is a drug effect occurring in certain individuals. It is different from the effects caused by genetic abnormalities. For example, morphine in most people causes depression effects, while in certain individuals, especially women, it can cause excitation [14]. DRP with the wrong drug subdomain (idiosyncrasies) was not found in our study group.

In 26 patients, DRPs were found, with indications that were not treated. We observed that the lack of treatment was due to the following three causes: New indications arise and previously chosen drugs were not prescribed; drugs were either not taken or administered; and despite the indications, drugs were not prescribed. Cases with indications that are not treated can be often found in non-DM conditions. Assessments are done based on laboratory data and specific details of the patient.

The patients' rapidly changing BSLs require routine monitoring and medications at specific intervals. We observed that missed routine monitoring is the primary cause for the lack of treatment in the DM patients included in our study group. Of note, one case was representative for many other DM cases. Specifically, the patient was admitted to the hospital with BSL (19.30 [9/3/15]), for which $46 \mathrm{mg} / \mathrm{dL}$ of D $40 \%$ IV bolus were administered along with a paired infusion of D10\% $15 \mathrm{gtt} / \mathrm{min}$. The DM drug was discontinued. BSL was examined at 2-time points - $23.00(9 / 3 / 15)$ with $107 \mathrm{mg} / \mathrm{dL}$ and 02.00 with $264 \mathrm{mg} / \mathrm{dL}$. Once the condition was cleared, no treatment for hyperglycemia was administered.

Another example of unaddressed indication is the case of a patient who was identified with a new indication; however, the drug to treat it was not prescribed. Based on our observations of unwritten admission diagnosis, the patient was anemic. Following a few days of treatment, the patients' blood work showed hemoglobin level of $9.9 \mathrm{~g} / \mathrm{dL}$ (normal value, $12-16 \mathrm{~g} / \mathrm{dL}$ ); however, no therapy was given to address the condition.

A number of indications remain untreated due to the failure of taking the drug or missed administration because the time of administration is not listed in the Kardex. For example, paracetamol was prescribed to a patient due to fever, but the time of administration of the drug was not listed in the Kardex.

Finally, we observed the case of a patient who was not treated despite the presence of indicators. Specifically, on admission, the patient underwent laboratory a checkup. Results showed a leukocyte value of $13,210 \mu \mathrm{L}$ (normal value: $4,800-10,800 \mu \mathrm{L}$ ). These results indicate the presence of an infection; however, no drug was prescribed.

\section{ADRs}

\section{Patients suffer ADR not allergies}

We observed 60 incidences of patients suffering from non-allergic ADR. ADR is not an allergy. It is caused by the combination of drugs, inappropriate medications, drug interactions, and the use of several drugs (different therapeutic group or active ingredients) prescribed for the same indication. ADR is often seen following the administration of ranitidine with a dose of $2 \times 2 \mathrm{ml}$ i.v. and omeprazole with a dose of $1 \times 40 \mathrm{mg}$ or in combination with other types of drugs such as sucralfate or rebamipide. Patients complain of abdominal pain; however, no further examination such as gastroscopy is performed to determine the condition of gastric or duodenal ulcers.

Yet when omeprazole is discontinued, the use of ranitidine alone sees the worsening of the gastric ulcers [15]. Another example can be observed in the case of the administration of drugs for hypertension. Common therapeutic approach consists of bisoprolol ( $\beta$-blocker and $\beta 1$-selective) and ramipril (ACE inhibitor). Specifically, a patient has a history of hypertension with blood pressure before the drug administration ranging between a systolic of 120 and $139 \mathrm{mmHg}$ and a diastolic of 80 and $89 \mathrm{mmHg}$. A potential UDR that may arise when treating this patient with both drugs is hypotension.

The potential for non-allergic ADR may be caused by the absence of an indication of drug use or indications of unclear drugs such as in the case of ranitidine or omeprazole and metoclopramide or ondansetron. Based on laboratory results or patient complaints, there is no indication whatsoever related to drug administration. UDR is possibly caused by ranitidine, in line with the condition of the patient, which is nausea, vomiting, and abdominal discomfort.

Patients with sudden hypoglycemia will have ADR resulting from too high doses. However, if the patient is still conscious, restoring the patients' blood glucose condition immediately by giving D40\% i.v. would result in the patients' BSL being too high $>250 \mathrm{mg} / \mathrm{dL}$. Selection of inappropriate drugs (i.e., drugs used not for the recommended indication) includes the use of contraindicated drugs that cause ADR. Examples of this instance are patients with hyperkalemia shown by laboratory tests, who were prescribed sustained-release potassium dosage form indicated for hypokalemia. Patients will continue to show hyperkalemia in the following days. Patients were given a D5\% infusion 
post-hypoglycemia; however, the patients' BSL was not monitored until it reached $403 \mathrm{mg} / \mathrm{dL}$. This case is an example of UDR due to the absence of a dose adjustment. This condition is classified as a subdomain causing deterioration/improvement of ill conditions requiring dose adjustment. Patients were administered Novorapid ${ }^{\circledR}$ with instructions to administer $3 \times 10 \mu$ at 6:00 am, 12:00, and 17:00 pm. Despite the first two injections afternoon BSL ( $55 \mathrm{mg} / \mathrm{dL}$ ), checks showed that patients were experiencing hypoglycemia. This patients' ADR was likely due to frequent dose regulation. The appropriate insulin dosage for patients weighing $40 \mathrm{~kg}$ ranges from 20 to $40 \mu$ /day.

We observed the improper timing of drug use or dosing interval potentially causing UDR following the administration of a prescribed antihypertensive drug ramipril $1 \times 1 \mathrm{mg}$. This drug was administered in conjunction with Novorapid $^{\circledR}$ at 06.00 , potentially causing hypoglycemia.

UDR in patients eating foods that interact with drugs was seen when drinking sugar beverages while hypoglycemic followed by the infusion of D $40 \%$ causing the patients' BSL to jump to $383 \mathrm{mg} / \mathrm{dL}$.

There were 37 actual or potential ADR incidences, which occurred due to drug interaction. Incidence of interaction between diabeticdiabetic drugs was $0.0 \%$, diabetic-non-diabetic drug interaction was $35.1 \%$, and non-diabetic-non-diabetic drug interaction was $64.9 \%$. An analysis performed with the Micromedex ${ }^{\circledR}$ software showed that non-oral (insulin)-non-diabetic drug interactions occurred between aspart insulin and the following drugs: ACE inhibitors (captopril and ramipril), which may cause hyperglycemia; quinolone antibiotics (levofloxacin), which may lead to increased risk of hypo/ hyperglycemia; $\beta$ blockers and $\beta 1$ selective (bisoprolol) lead to hypo/hyperglycemia and hypertension; and angiotensin II receptor blocker, which increases the risk of hypoglycemia. Potential UDR problems also occurred due to the interaction of hypoglycemic oral diabetic-non-diabetic drugs between sulfonylurea (metformin) and ACE inhibitor (ramipril) drugs. Specifically, the administration of both of these drugs simultaneously causes excessive hypoglycemia. The most common non-diabetic group of drugs that cause potential drug interactions among non-diabetics is antibiotics and ACE inhibitors. Antibiotics are a common cause of moderate-to-severe drug interactions $[13,16]$.

\section{Patients suffering from ADR allergy}

$\mathrm{ADR}$ is a direct danger arising from normal drug also when using normal doses. Allergies are undesirable drug reactions mediated by the immune system. Treatment of a mild allergic reaction required discontinuation of the drug. On the contrary, for moderate-to-severe allergic reactions, assessment and treatment are necessary. Unsolicited drug reactions of severe allergies can lead to disability and even death. In our study, we have not observed the provision of drugs at risk of causing ADR allergy.

\section{Patients suffering from toxic effects}

Drug use will always pose a risk even if it is kept to a minimum. This is due to possible infrequent toxic reactions or side effects. All chemicals are toxic, and poisoning is determined by the dose and mode of administration [17]. DRP with a subset of patients suffering from potentially toxic effects occurred in 22 of the observed cases. Five possible causes of potentially toxic effects have been identified: Frequent dose regulation; pharmacokinetics-related problems requiring dose adjustment; inappropriate drug use/dose interval times; excessively high doses; and prescribing errors (in terms of drug prescription).

An excessively frequent dose regulation was found in omeprazole drug administration. We observed that omeprazole dosage was $2 \times 40 \mathrm{mg}$ i.v. However, the dosage should be $1 \times 20-40 \mathrm{mg}$. It is also known that overdose/toxic events associated with this drug are minimal, with hypotension as the most common symptom.

Non-DM drugs caused the majority of toxic effects. This relates to pharmacokinetics-related problems that required dose adjustment and inappropriate drug use/interval time. There should be a dose adjustment for renal impairment. Calculation of creatinine clearance, using the Cockcroft and Gault method, found that patients with diabetic nephropathy complications had clearance $<50 \mathrm{ml} / \mathrm{min}$. In these cases, a dosage adjustment was required, administering $50 \mathrm{mg}$ of ranitidine every $18-24 \mathrm{~h}$ as well as metoclopramide $25 \%$ of normal dose given in case of clearance $<10 \mathrm{ml} / \mathrm{min}$. An example of this occurrence was a 77-year-old patient with serum creatinine $3.4 \mathrm{mg} / \mathrm{dL}$, creatinine clearance $9.26 \mathrm{ml} / \mathrm{min}$, and $40 \mathrm{~kg}$ body weight.

Kidney damage due to DM affects the pharmacokinetics of the drug. Acute illness or kidney trauma will reduce glomerular filtration and/or active secretion, causing a longer elimination time of the drug, leading to a potential drug accumulation in the body and higher risk of toxic effects [17].

DRP caused by toxic effects of drugs was also found following ranitidine administration in patients suffering from diabetic nephropathy. For these patients, the prescription required ranitidine administration $2 \times 50 \mathrm{mg}$ i.v. without any clear dose interval. In general, ranitidine administered in the cardiac is in intervals of 7-8 h.

Prescription errors (in the case of prescriptions) that have the potential to cause toxic effects in patients were seen with the prescription of folic acid $3 \times 50 \mathrm{mg}$. Excessive folic acid can cause progressive nerve damage.

\section{CONCLUSION}

In this study, the percentage of DRP occurring amounted to $50.6 \%$ of therapeutic effectiveness problems and $49.4 \%$ ADR. Non-allergic ADR is a DRP subdomain that has the greatest frequency of occurrence (36.1\%).

\section{CONFLICTS OF INTEREST}

The authors have no conflicts of interest.

\section{REFERENCES}

1. Indonesian Ministry of Health. Directorate of Community and Clinical Pharmacy Development, Directorate General of Pharmaceutical and Medical Devices, Indonesian Ministry of Health. Pharmaceutical Care for Diabetes Mellitus. Jakarta: Indonesian Ministry of Health; 2005.

2. DiPiro JT, Talbert RL, Yee G, Matzke G, Wells B, Posey LM. Pharmacotherapy: A Pathophysiologic Approach. $7^{\text {th }}$ ed. United States of America: McGraw-Hill; 2008.

3. Sievenpiper JL, Dworatzek PD. Food and dietary pattern-based recommendations: An emerging approach to clinical practice guidelines for nutrition therapy in diabetes. Can J Diabetes 2013;37:51-7.

4. Dennedy MC. Management of Type 1 diabetes mellitus. Medicine 2010;38:610-7.

5. Pharmaceutical Care Network Europe Foundation. PCNE Classification for Drug related problems (revised 14-01-2010 vm) V6.2. Netherland: Pharmaceutical Care Network Europe Foundation; 2010.

6. Indonesian Ministry of Health. Directorate of Community and Clinical Pharmacy Development, Directorate General of Pharmaceutical and Medical Devices, Indonesian Ministry of Health. Guideline on Drugs Therapy Monitoring. Jakarta: Indonesian Ministry of Health; 2009.

7. Semla TP, Rochon PA, Cobbs EL, Duthie EH, Murphy JB. Pharmacotherapy. In: Geriatrics Review Syllabus: A Core Curriculum in Geriatric Medicine. Boston: Blackwell; 2005.

8. Hanlon JT, Schmader KE, Ruby CM, Weinberger M. Suboptimal prescribing in older inpatients and outpatients. J Am Geriatr Soc 2001;49:200-9.

9. Fulton MM, Allen ER. Polypharmacy in the elderly: A literature review. J Am Acad Nurse Pract 2005;17:123-32.

10. Hajjar ER, Cafiero AC, Hanlon JT. Polypharmacy in elderly patients. Am J Geriatr Pharmacother 2007;5:345-51.

11. Björkman IK, Sanner MA, Bernsten CB. Comparing 4 classification systems for drug-related problems: Processes and functions. Res Social 
Adm Pharm 2008;4:320-31.

12. Indonesian Ministry of Health. Directorate of Community and Clinical Pharmacy Development, Directorate General of Pharmaceutical and Medical Devices, Indonesian Ministry of Health. Guideline on Visit. Jakarta: Indonesian Ministry of Health; 2011.

13. Wright J. Complications of antibiotic therapy. Med Clin North Am 2013;97:356420.

14. Pawitan JA. Nerve and Mental Health Module. Jakarta: Faculty of Medicine Universitas Indonesia; 2007.
15. Lester GD, Smith RL, Robertson ID. Effect of treatment with omeprazole or ranitidine on gastric squamous ulceration in racing through breds. J Am Vet Med Assoc 2005;227:1636-9.

16. Lai L, Gharib S. Pcv65 drug-drug interactions on angiotensin converting enzyme (ace) inhibitor prescriptions in the United States: 2001 to 2004. Value Health 2007;10:A55-6.

17. Shargel L, Wu-Pong Susanna BC, Yu A. Applied Biopharmaceutics and Pharmakokinetics. New York: The McGraw-Hill Companies, Inc; 2005 . 